\title{
Gestion de l'environnement pour les bassins hydrominéraux de Contrexeville et de Vittel
}

\author{
J. Teysseyre
}

Nestlé Sources International - Site Est - Vittel

\section{LES BASSINS HYDROMINÉRAUX DE CONTREX ET DE VITTEL}

Dans la région occidentale du département des Vosges, les bassins hydrominéraux de Contrex et de Vittel sont constitués en surface par des plateaux calcaires et marnocalcaires de 12000 ha environ, qui descendent en pente douce vers le Nord-Ouest.

Le climat est continental et la pluviométrie abondante : 900 millimètres d'eau en moyenne par an. Une partie de ces eaux contribue au renouvellement des nappes hydrominérales en traversant les formations sédimentaires du trias, appuyées sur un socle granitique.

Trois gîtes hydrominéraux sont exploités :

- le gîte supérieur: localisé dans le Keuper inférieur et la Lettenkohle,

- le gîte moyen, séparé du gîte supérieur par la série marnocalcaire du Muschelkalk supérieur, est capté dans le Muschelkalk moyen,

- le gîte inférieur est séparé du gîte moyen par des argiles bariolées à la base du Muschelkalk moyen. Il est capté dans les grès du trias inférieur (grès vosgiens).

\section{II — PRÉSENTATION DES ÉCOSYSTÈMES}

En 1860 pour Contrex et 1903 pour Vittel, les eaux minérales ont été déclarées d'intérêt public et bénéficient de périmètres de protection. Les nombreuses modifications de périmètres trouvant leurs bases dans les études hydrogéologiques, ont permis d'adapter précisément leurs limites aux bassins d'alimentation.

La préservation des écosystèmes, que représentent ces bassins, est nécessaire pour garantir sur un long terme la constance de la qualité des eaux minérales.

Les différentes activités humaines peuvent être sources de nuisances, qu'elles soient :

- urbaines, industrielles, artisanales,

- transports et stockage de matières combustibles ou dangereuses,

- agricoles.

Il convient donc de connaître ces différentes activités afin de prévenir au maximum les différents risques pour les nappes hydrominérales.

\section{MÉTHODOLOGIE DE TRAVAIL}

Pourquoi ? Où ? Comment ? intervenir utilement en matière de protection des écosystèmes.

Depuis 1975, un réseau d'observations a été mis en place sur les eaux de surfaces et de subsurfaces (sourcettes, ruisseaux). Il est réactualisé régulièrement. L'analyse de risques pour l'ensemble des activités humaines sur les bassins a été effectuée à partir de la description détaillée

\section{Environmental management for the mineral water basins of Contrexeville and Vittel}

Guaranteeing a constant water quality is a primary requirement. This involves preserving the ecosystem that forms the mineral water basin.

Protection of the mineral water system has its origins in the history of the company with the first work being carried out at the beginning of the century.

This protection approach is based upon a great deal of hydrogeological research: it has involved a number of actions concerned with the various human activities such as urbanisation, craft trades, industry and, more particularly, agriculture.

As far as farmers are concerned, we are encouraging them to introduce new cropping schemes whereby a specification can be met. To facilitate these changes, we are making use of technical and financial aid schemes and are implementing $a$ land purchase policy. 
des produits mis en œuvre ainsi que des rejets et des résultats du réseau d'observations. Pour chacun des risques, des objectifs de travail ont été fixés et des moyens mis en place.

\section{IV —URBANISATION ET PRÉSERVATION DES ÉCOSYSTÈMES}

\subsection{Eaux usées}

Dès 1912, la ville de Vittel a réalisé la collecte des eaux usées. Une station d'épuration, très performante pour l'époque, a traité ces eaux depuis la fin des années 1920. Parallèlement, la ville de Contrex a mis en place son propre réseau.

Depuis 1971 , les autres communes faisant partie intégrante des bassins ont vu leurs eaux usées collectées et envoyées dans la station d'épuration existante. Vittel a pris en charge l'assainissement de ces communes (réseaux de collecte et accompagnement jusqu'à la station d'épuration).

1994 a vu le démarrage d'une nouvelle station d'épuration destinée à traiter les eaux usées des communes et des installations industrielles. Cette station d'épuration a pu être réalisée grâce à la constitution d'un SIVU regroupant les municipalités de Contrex et de Vittel et les deux sociétés d'eaux minérales. Le procédé de traitement de cette station d'épuration a été particulièrement étudié et permet de respecter des normes de rejets drastiques par exemple moins de $1 \mathrm{mg} / \mathrm{l}$ de phosphore total.

\subsection{Autres déchets}

D'autres rejets urbains doivent être éliminés. Les municipalités ont mis en place plusieurs systèmes de collectes, depuis les plus classiques (ordures, emballages) jusqu'à d'autres plus originaux (produits chimiques, médicaments, piles, etc...).

Les artisans, par leurs activités, génèrent des déchets. Là encore une collecte spécifique a été mise en place: elle concerne les huiles usagées pour les garages et les solvants utilisés pour les artisans peintres et carrossiers. La mise en place de cette collecte a pu se faire grâce à la collaboration des artisans, des municipalités, des sociétés de traitement, des deux sociétés d'embouteillage. L'exemplarité de cette collecte a permis que l'ensemble des frais soit réparti entre les différents partenaires.

\section{COMBUSTIBLES, MATIÈRES DANGE- REUSES ET PRÉSERVATION DES ÉCO- SYSTÈMES}

Dans ce cas, les risques environnementaux sont liés :

- aux produits eux-mêmes et à leurs stockages,

- aux transports des produits.

\subsection{Les produits}

Les citernes de fuel, dont certaines enterrées dans les années 50 représentent un risque potentiel. De plus, pour contribuer à la réduction de la pollution atmosphérique, à la fin des années 1980, les établissements industriels ont changé de combustibles, passant du fuel lourd au gaz de G.D.F. Ce faisant, la région a pu être raccordée au réseau et chaque particulier s'est vu offrir la possibilité de remplacer le fuel par le gaz. Une collaboration CommunesSociétés d'eaux minérales-G.D.F. a permis de promouvoir le Gaz. Chacun des partenaires aide au financement du changement de combustible ; les sociétés assurent gratuitement le dégazage du réservoir ancien à fuel et son enlèvement dans la mesure du possible, ou son inertage.

\subsection{Le transport des produits}

D'une part, pour pallier à tout risque de pollution en cas d'accident mettant en cause des véhicules transportant des produits dangereux, les municipalités et les sociétés d'eaux minérales se sont associées pour doter le centre de secours d'un véhicule de dépollution.

D'autre part, afin de faciliter la circulation, une déviation de Vittel est en cours de construction. Les services de la D.D.E., avec la participation de VITTEL SA, ont étudié et mis en place des moyens permettant la collecte et la maîtrise de tous les déversements accidentels de produits dangereux et des eaux de ruissellement.

La protection optimale, décrite dans un Arrêté Ministériel, passe par l'étanchéification de la chaussée, mais aussi des fossés et des bassins de récupération. Ces bassins permettent d'intercepter le ruissellement, de le traiter et enfin de l'évacuer.

Des clauses particulières des pièces contractuelles des marchés stipulent que les entreprises doivent se conformer à des prescriptions précises. Parmi celles-ci on peut citer :

- les stockages des carburants et l'entretien des matériels doivent se faire hors du périmètre de protection,

- les plates-formes étanches pour le parcage momentané des engins,

- l'évacuation en cours de chantier des eaux de ruissellement,

- aucune fuite d'huile hydraulique n'est acceptée sur les engins de chantier.

Enfin, pour s'assurer du respect de toutes les mesures prises, un hydrogéologue suit de façon continue l'ensemble de tous les travaux.

\section{AGRICULTURE ET PRÉSERVATION DES ÉCOSYSTÈMES}

Dès les années 70, notre attention se portait, d'une façon générale, sur l'évolution des nitrates dans les eaux en France. Aussi, une première étude d'évaluation des risques était engagée sur le bassin de Vittel en 1975 avec l'ENSAIA. Ses conclusions tendaient à montrer que :

- les risques agricoles étaient réduits,

- l'apparition de la culture du maïs sur la zone, constituait un fait nouveau. 
Conscient du risque, même faible, on a pris la décision de constituer un réseau de surveillance important des eaux de ruissellement et des eaux de subsurfaces à cette même date. Dès les années 80 , on décidait d'agir préventivement. Les premiers contacts étaient alors pris avec le monde agricole.

\subsection{Etude par I'INRA}

En 1989, une équipe de recherche pluridisciplinaire était constituée (agronomes, zootechniciens, géographes, pédologues, économistes, sociologues, historiens) avec comme sujet de recherche ambitieux :

«Comment maintenir une agriculture performante tout en préservant la qualité des eaux minérales ». Cette étude étāit cofinancée par l'Agence de Bassin Rhin-Meuse, I'INRA et VITTEL S.A.

Après 3 ans d'études, l'INRA a préconisé de nouvelles pratiques culturales, vérifié sur trois exploitations témoins le bien-fondé de ces pratiques, ceci d'un point de vue technique mais aussi économique. Enfin, l'incidence sociologique sur l'ensemble des bassins a été précisée.

L'INRA a pu proposer un cahier des charges permettant de cultiver les terres des bassins en mettant en œuvre des pratiques culturales préservant la qualité des eaux minérales. Il repose sur 7 points essentiels :

1) supprimer la culture du maïs,

2) composter les déjections animales et apport d'azote raisonné,

3) zéro produit phytosanitaire,

4) chargement à l'ha ne dépassant pas 1 UGB (Unité de gros bétail) de SFP (Surface fourragère principale),

5) mise en place de rotations à base de luzerne,

6) équilibrer la ration animale,

7) mettre aux normes les bâtiments d'exploitation.

Cette étude est actuellement poursuivie ; elle permet de préciser dans le détail l'ensemble des solutions choisies et la mise en place des mesures d'accompagnement.

\subsection{Cahier des charges et agriculteurs}

Depuis deux ans, ces nouvelles pratiques culturales sont expliquées aux agriculteurs par l'INRA et VITTEL SA. Aujourd'hui, les agriculteurs travaillant $60 \%$ des 3400 ha de terres cultivées du bassin hydrominéral de Vittel, ont signé une convention avec VITTEL SA par laquelle ils s'engagent à appliquer le cahier des charges.

\subsection{Aides au changement}

Les sociétés d'eaux minérales ont fixé pour objectif que les revenus agricoles résultant des nouvelles pratiques, soient au moins égaux à ceux des anciennes cultures. Afin de faciliter le passage des anciennes aux nouvelles pratiques, des aides temporaires sont proposées aux agriculteurs.

\subsubsection{Aides générales}

Une aide générale de $1200 \mathrm{~F} / \mathrm{ha}$ sur 5 ans est donnée à tout agriculteur acceptant de mettre en place le cahier des charges sur la totalité de son exploitation.

Ces aides sont financées par trois opérateurs :

- les municipalités de Contrex et de Vittel pour un montant de $600 \mathrm{~F} / \mathrm{ha}$,

- le ministère de l'agriculture pour $300 \mathrm{~F} / \mathrm{ha}$ jugeant l'intérêt de la démarche et l'exemplarité des études effectuées,

— les deux sociétés d'eaux minérales, pour 300 F/ha.

\subsubsection{Aides particulières}

Les sociétés se sont engagées à compléter ces aides générales. Ce complément est modulé en fonction de l'entreprise agricole, il peut être proposé tout ou partie de :

— un complément d'aides financières,

- une aide aux investissements :

- réalisation d'une aire de compostage des déjections animales, ainsi que le compostage et l'épandage,

- réalisation d'un bâtiment de séchage en grange du fourrage,

- financement d'une nouvelle chaîne de récolte adaptée à l'herbe et comprenant une faucheuse et une autochargeuse,

- participation au financement de la mise aux normes des bâtiments d'exploitation, en compléments aux aides de l'Agence de Bassin et du Conseil Régional.

- une aide sous forme de services ou de prêts de matériels :

- compostage des déjections animales et l'épandage :

- prêt de matériels spécifiques: herse, bineusedésherbeuse, déchaumeur,

- formation aux nouvelles pratiques.

\subsection{Achats de terres}

A partir de 1989, VITTEL SA a proposé le rachat de terres libres à la vente à l'intérieur du périmètre. Il a été acquis à ce jour 1400 ha sur les 3400 ha de terres cultivées sur ce bassin. Ces terres sont remises à la disposition d'agriculteurs à la condition qu'ils acceptent de respecter le cahier des charges sur la totalité de leur exploitation. L'acquisition des terres et leur redistribution se font par l'intermédiaire d'un opérateur foncier: la SAFER LORRAINE.

Le système de gestion est le suivant :

- distribution de terres aux agriculteurs, sous forme de convention d'occupation précaire, avec obligation de mise en herbe, apport d'azote raisonné et zéro produit phytosanitaire,

— signature d'un bail emphytéotique VITTEL-SAFER, - signature de baux emphytéotiques SAFER-agriculteurs portant sur les terres propriétés de Vittel, avec obligation, pour l'agriculteur, d'appliquer le cahier des charges sur l'ensemble des terres de son exploitation. 


\subsection{Modes de cultures des golfs et parcs}

600 ha constituent la zone touristique ; elle est essentiellement constituée de parcs et de golfs dans lesquels l'esthétique joue un rôle important.

Le cahier des charges de l'INRA a été appliqué en 1994, pour la $3^{\mathrm{e}}$ année consécutive. Les contraintes pour ces cultures sont identiques à celles demandées dans le monde agricole.

Cependant des modes de cultures spécifiques ont dû être développés :

- abandon des herbicides et utilisation du désherbage thermique (on peut d'ailleurs signaler la signature d'une convention SNCF-VITTEL permettant l'application du désherbage thermique sur les 35 ha des voies ferrées),

- sélection de porte-greffes et de variétés de rosiers résistants aux maladies et adaptés à notre climat (abandon des pesticides),

- maîtrise de pratiques préventives (brossage des arbres, ramassage systématique des feuilles),

- maitrise du développement de parasites (pucerons : larves de coccinelles, mulots : rapaces).

\subsection{Les arbres, les haies et la forêt}

Les arbres, les haies et la forêt jouent un rôle important dans les écosystèmes constituant les bassins hydrominéraux. En effet ils permettent de :

- réguler le régime des eaux de ruissellement et diminuer les risques d'érosion,
- diminuer les risques sanitaires en freinant la dissémination des maladies cryptogamiques et des insectes nuisibles,

- d'augmenter la population des insectes auxiliaires et des prédateurs permettant de contrôler la population de nuisibles,

- d'améliorer l'esthétique des paysages.

Il est donc important de maintenir et même de développer l'arbre. Sur les deux bassins hydrominéraux, la surface boisée représente actuellement environ $30 \%$.

Une étude menée avec l'O.N.F. doit permettre de déboucher fin 1994 sur la proposition d'un projet de reboisement (essences, densités).

Ce test permettra d'envisager une gestion efficace des haies, du boisement et des ripisylves.

\section{VII $\square$ CONCLUSION}

La coopération et la contribution des nombreux intervenants sur les bassins (publics et privés) ont permis de mobiliser les forces pour modifier l'esprit et les méthodes de travail, ceci dans le but d'assurer la pérennité des eaux minérales des bassins de Contrex et de Vittel.

Ces personnes ont constamment présente à l'esprit la phrase d'Antoine de Saint-Exupéry:

« Nous ne sommes pas les héritiers de nos parents, mais les locataires de nos enfants ». 